\title{
Validation study of STOP-Bang score for screening sleep-disordered breathing
}

\author{
Tomoyuki Kawada ${ }^{1}$
}

Received: 12 January 2016/Accepted: 14 March 2016 / Published online: 24 March 2016

(C) Springer-Verlag Berlin Heidelberg 2016

\section{Dear Editor,}

Nahapetian et al. conducted a validation study of three variations on STOP-Bang score for sleepdisordered breathing (SDB) [1]. The authors calculated conventional, weighted, and continuous STOP-Bang scores, and compared with apnea-hypopnea index (AHI) $>15 / \mathrm{h}$ by in-home polysomnograms-derived SDB. Receiver operating characteristic (ROC) curves analysis was adopted for the screening of SDB. I have two queries on their study outcome.

First, the authors concluded that modified STOP-Bang score such as weighting the variables and using continuous variables of body mass index (BMI), age, and neck circumference had an advantage against conventional STOP-Bang score. But the authors also described that area under the curve (AUC) of continuous STOP-Bang score was significantly larger than those of conventional and weighting STOP-Bang score. Please describe the consistent results. Unfortunately, there was no description of statistical method for comparing two AUCs in their Table 4 and Figure 1. The ROC curve analysis was first introduced in Radiology [2-4], and the size of the sample is important for the statistical power. Although I appreciate the enough number of samples in their analysis, please specify the name of the statistical software for conducting statistical comparison of AUC in each ROC curve.

Second, the authors adopted BMI $>35$ as the cut-off value of SDB. Kim et al. adopted BMI $>30$ as the cut-off value for

Tomoyuki Kawada

kawada@nms.ac.jp

1 Department of Hygiene and Public Health, Nippon Medical School, 1-1-5 Sendagi, Bunkyo-Ku, Tokyo 113-8602, Japan
Korean subjects and concluded that (conventional) STOPBang questionnaire kept high sensitivity for screening obstructive sleep apnea [5]. But its specificity was lower than the acceptable level, when compared with the Sleep Apnea of Sleep Disorder Questionnaire. There is a difference in the distribution of BMI in subjects with different ethnicity. I suppose that neck circumference should also be re-evaluated in subjects with different ethnicity. Taken together, the criteria of conventional STOP-Bang score can be applied to a population within the same ethnicity.

\section{Compliance with ethical standards}

Disclosure The author has indicated no financial support.

Conflicts of interest The author declares no conflict of interest.

\section{References}

1. Nahapetian R, Silva GE, Vana KD, Parthasarathy S, Quan SF (2015) Weighted STOP-Bang and screening for sleepdisordered breathing. Sleep Breath. doi:10.1007/s11325-015$1255-2$

2. Hanley JA, McNeil BJ (1982) The meaning and use of the area under a receiver operating characteristic (ROC) curve. Radiology 143:29-36

3. Hanley JA, McNeil BJ (1983) A method of comparing the areas under receiver operating characteristic curves derived from the same cases. Radiology 148:839-843

4. McNeil BJ, Hanley JA, Funkenstein HH, Wallman J (1983) Paired receiver operating characteristic curves and the effect of history on radiographic interpretation. CT of the head as a case study. Radiology 149:75-77

5. Kim B, Lee EM, Chung YS, Kim WS, Lee SA (2015) The utility of three screening questionnaires for obstructive sleep apnea in a sleep clinic setting. Yonsei Med J 56:684-690 